\title{
IC-Based Rectification Circuit Techniques for Biomedical Energy-Harvesting Applications
}

\author{
Cihun-Siyong Alex Gong 1,2,3 \\ 1 Department of Electrical Engineering, School of Electrical and Computer Engineering, College of Engineering, \\ Chang Gung University, Taoyuan 33302, Taiwan; alexgong@mail.cgu.edu.tw \\ 2 Portable Energy System Group, Green Technology Research Center, College of Engineering, \\ Chang Gung University, Taoyuan 33302, Taiwan \\ 3 Department of Ophthalmology, Chang Gung Memorial Hospital, Linkou, Taoyuan 33305, Taiwan
}

Citation: Gong, C.-S.A. IC-Based Rectification Circuit Techniques for Biomedical Energy-Harvesting Applications. Micromachines 2022, 13, 411. https://doi.org/10.3390/ mi13030411

Academic Editors: Nam-Trung Nguyen, Haixia (Alice) Zhang and Daewon Kim

Received: 26 January 2022

Accepted: 3 March 2022

Published: 5 March 2022

Publisher's Note: MDPI stays neutral with regard to jurisdictional claims in published maps and institutional affiliations.

Copyright: (C) 2022 by the author Licensee MDPI, Basel, Switzerland. This article is an open access article distributed under the terms and conditions of the Creative Commons Attribution (CC BY) license (https:/ / creativecommons.org/licenses/by/ $4.0 /)$.

\begin{abstract}
Energy harvesting can be achieved through many different mechanisms. Such technology has been drawing researchers' attention to its practical applications for a decade, as it can be widely applied to countless scenarios. It steals the show in the modern development of the biomedical electronics, especially implantable applications, as it allows the patients to move freely without restriction. To prolong lifetime of the battery inside/outside a patient's body, the electrical conversion efficiency of the electronic implant is of primary importance in energy harvesting. The conversion can be achieved by a so-called miniaturized rectification circuit (also known as "rectifier"). This study aims to compare different state-of-the-art techniques focusing on the conversion efficiency of the rectification. Particular emphasis is put on semiconductor-based circuits capable of being integrated with tiny chips on the implants.
\end{abstract}

Keywords: biomedical; implantable; prostheses; biomedical; AC-DC; energy harvesting; conversion efficiency; rectifier

\section{Introduction}

Current IC design and foundry processes increasingly allow for the manufacture of sophisticated system-on-chip (SoC) applications, allowing for the miniaturization of integrated biomedical devices. Implantable medical chips (also referred to as "implantable microelectronics") are cross-disciplinary integrated systems which have advanced rapidly over the past half year, producing a string of new applications for the treatment of many major neurological and physiological conditions related to visual, auditory, neuromuscular, neurological or other functions, thus offering the possibility of tremendous improvements to quality of life.

Looking ahead, health-related technologies have emerged as the focus for the next generation of industrial development, and are sure to have a far-reaching impact on human health and quality of life. To promote the development of such technologies, countries around the world are actively establishing cross-disciplinary medical research groups. Currently, applications using implantable chips allow surgeons to diagnose and formulate treatment options for brain lesions in neuropathy patients [1], while high-performance implanted chips are being developed for use in artificial ear [2] and eye [3] applications.

Figure 1 shows the implantable applications. Pacemakers are used to assist defective hearts and correct arrhythmia. When the heart beats irregularly, the pacemaker emits electrical pulses to restore the correct rhythm. Artificial ears are used to restore auditory sensitivity to patients suffering from partial or total hearing loss, with external radio components sending electronic signals and electrical power through a wireless coupling to the implanted components which reproduce external sounds in the inner ear, thus restoring hearing function. Retinal electronic implants are used to restore visual function. 
Unfortunately, current battery technology has not yet been able to support the permanent operation of them, and depleted batteries used in the implanted devices must be replaced.

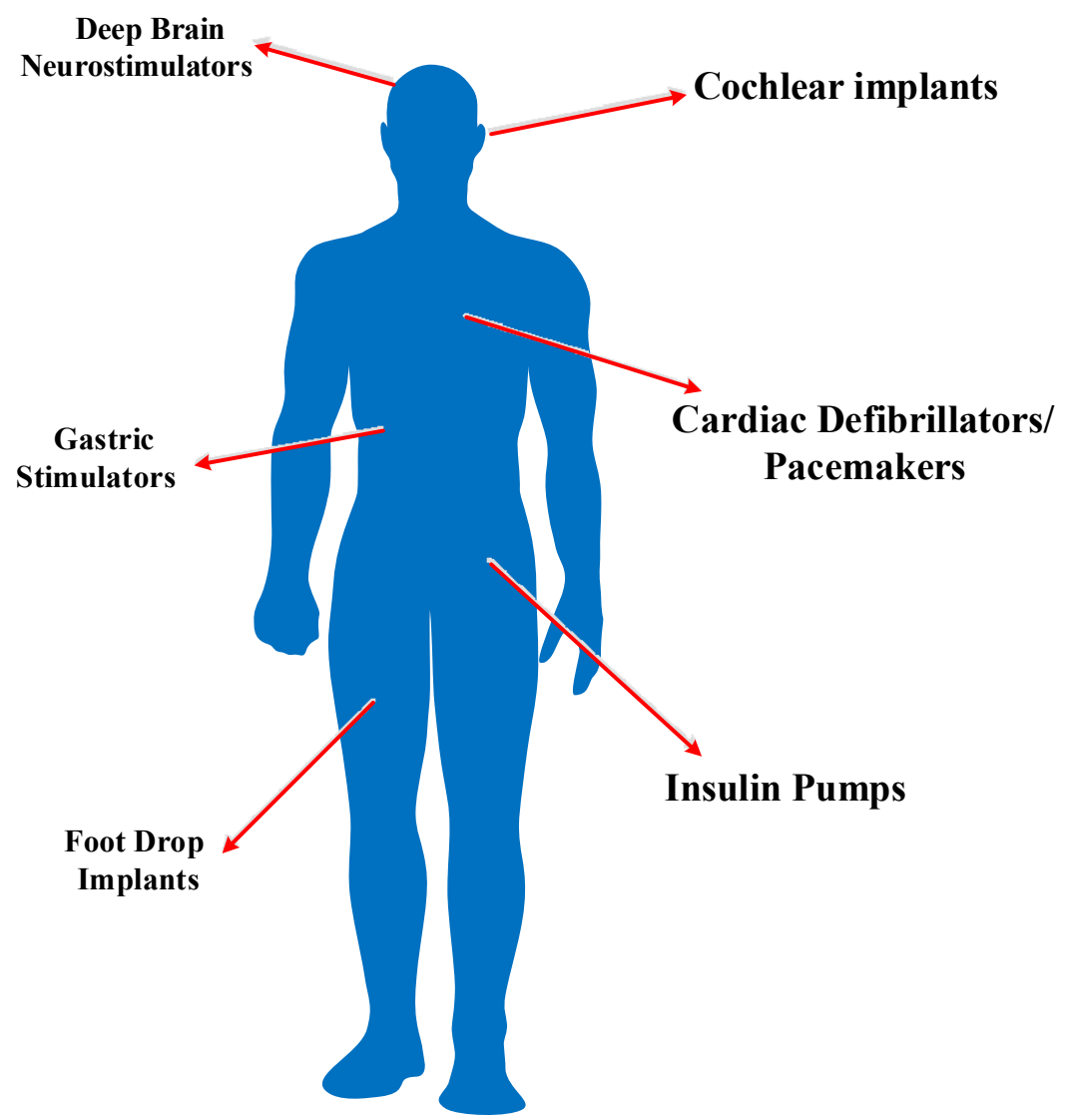

Figure 1. Implantable medical devices for various applications.

Wireless Implantable Medical Systems

Radio power transmission technology has been proposed to address the lifetime problem. Taking the Retinal Electronic Prostheses (REPs) as an example, the system in Figure 2 consists of a sensor interface external to the eyeball, used to capture images and other signals (not on the PC shown in the image). All captured signals are processed using parameters determined by the clinician. These parameters determine the pulse duration, stimulation time and output duration (i.e., the width of the current pulse) when the module is engaged. A series of signal processing steps follows, including coding and modulation to obtain information required for generating visual perception. This information will determine the size of the injected current generated by the microstimulator module for electrical neuronal stimulation. Finally, the integrated signal is amplified by the external platform and transmitted in vivo.

The electrodes made by MEMS technology are closely connected to the electrical stimulator and the neural signal recording circuit, and the process technology and material used will greatly affect the electrical driving ability and the clarity of the signal recording. Different electrode sizes and electrode materials will directly affect the electrode impedance, noise, and charge tolerance. Depending on the application and stimulation waveform, adjustment of electrode size and electrode spacing can significantly affect the physiological response. In addition, MEMS packaging also determines long-term success or failure of the chip.

Implantable systems use inductive coupling for reception through the built-in antenna module for the sake of chronic implantation, which consists of two parts. The first receives the carrier signal through alternating AC-DC and DC-DC modules to provide a stable power supply for the active circuit. At the same time, the processed data from the external 
platform are transmitted to the in vivo system for demodulation by a component which simultaneously generates a time signal for the in vivo system. Compared to approaches which supply power through a wire connected directly to the eye or that positions batteries within the eye, this power supply approach allows for perpetual use following a single operation to implant the "System on a Chip, SOC", while also eliminating the risk of battery leakage or infection [4].

The stimulator activates at the appropriate time based on the implant demodulation and subsequent data processing. Then, the signal passes through the pre-amplifier to record the neural response, which is produced when the stimulus current reaches the activation threshold. The recorded neural response can be modulated by the on-implant modulator for external transmission. The modulating module also allows the in vitro platform to receive the recorded neural signal for analysis by the external platform (shown here as a PC) to assess implant performance and study physiological signals through the SOC. This paper primarily focuses on the AC-DC module.

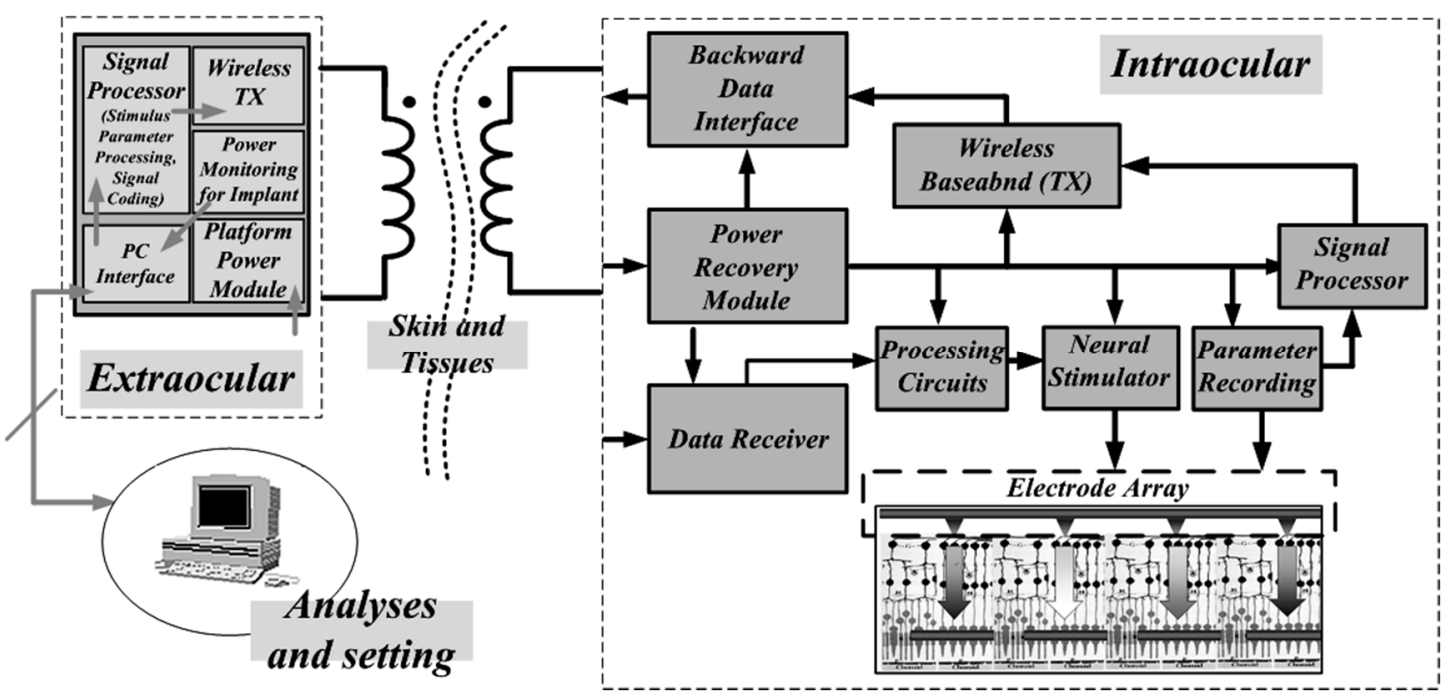

Figure 2. Wireless implantable system architecture for a Retinal Electronic Prosthesis with an external PC used to analyze various physiological and environmental parameters taken from intraocular sensor records.

\section{Underlying Principles of Wireless Powering}

\subsection{Theory}

Wireless energy transfer mainly consists of two antennas with an electromagnetic field (EM) transferring LC resonance energy between panel A and panel B. Wireless energy transmission can be basically classified as Near-Field Communication (NFC) and Far-Field Communication (FFC), where NFC is further divided into the Non-Radiative (Reactive) and Radiative (Fresnel). The key difference between these are the distance and linear dimension of the antenna. In Far Field, the electromagnetic waves are completely free from transmitter interference. The energy is transmitted over a long distance by electromagnetic waves, and can be described by the Friss Formula. Near Field can be divided into Reactive and Fresnel types. Reactive Near Field requires a much larger component than Fresnel, meaning any change in the electrical characteristics of the electrical field antenna or the magnetic characteristics of the magnetic field will have a significant impact on the impedance of the antenna input point. Fresnel Near Field is dominated by the Fresnel field, and the surrounding medium has little impact on antenna impedance. However, the distance between this area and the antenna is very small, so the antenna size is a factor that must be considered, thus the angular distribution of the Fresnel pattern is related to the distance. Overall, the characteristics of Near Field are that it has a short electromagnetic wave transmission range, but with a large energy volume and relatively small path loss. When 
setting conditions to limit a specific absorption rate, it can provide smaller loss than Far Field. For transmitting energy and information, these characteristics show that Far Field provides safer battery use conditions, and thus is typically used for wireless energy transmission for implanted biomedical devices.

\subsection{Frequency Selection}

From this discussion, we see that most NFC and FFC solutions use NFC for transmission in biomedical applications. Whether for RFID wireless communication of wireless charging in biomedical applications, there are strict specifications for carrier frequency and frequency bands, mainly to prevent mutual interference between wireless products. Therefore, it is necessary to formulate specifications to ensure that the frequencies used by various products will not cause interference. Most restricted frequency bands are not open, and the vast majority are restricted to commercial or military use. For example, commercial use frequencies are sold by auction to commercial bidders, while military use frequencies are allocated by governments based on national defense considerations. Some limited frequency bands are open to unrestricted public use under certain conditions, such as those developed by the International Telecommunications Union Radiocommunications Sector (ITU-R), or the Industrial, Scientific and Medical band (ISM) which is a frequency band limited for use by industrial, scientific and medical institutions.

Use of these frequencies does not require specific licensing or fees, but usage applications must comply with certain transmission power limits (generally under $1 \mathrm{~W}$ ) and must avoid causing interference with other frequencies. For example, $2.45 \mathrm{GHz}$ is the high-frequency oscillation band commonly used in microwave ovens, wireless network systems (e.g., mobile phone networks, Bluetooth, satellite TV and wireless local area networks, etc.), and sensor systems. In addition, $13.56 \mathrm{MHz}$ is currently the most widely used frequency band for industry, science and medicine, thus most wireless power modules are designed to use this band, but other frequency bands must also be used depending on biomedicine application specifications. In principle, the higher the frequency band, the smaller the distance attenuation. Medical applications should not exceed $20 \mathrm{MHZ}$ because frequencies above this range can be absorbed by human cells and have potentially negative health effects.

\subsection{Wireless Powering in Medicine}

Most biomedical systems transmit in a range of approximately $100 \mathrm{kHz}$ to $20 \mathrm{MHz}$. Wireless energy transmission in this frequency band not only meets the requirement for wireless charging, but also provides telemetry recursive data transmission, but this is limited to the ISM Band in Table 1 Most current commercial wireless transmission specifications are still based on $6.78 \mathrm{MHz}$ and $13.56 \mathrm{MHz}$, further promoted by mutual agreement between the WPC, A4WP and PMA (Table 1), thus the following sections focus on these two frequency bands and higher.

Electromagnetic induction refers to a conductor in a changing magnetic flux that generates an induced voltage called an induced voltage. If this conductor is closed into a loop, the voltage drives a flow of electrons, forming an induced current. The advantage of this approach is its low cost, while the disadvantage is that the magnetic material is not bound, and the magnetic flux of the transmitting coil will not pass through the receiving coil, thus it is necessary to closely align the wireless charger and the charging object. At present, the development of inductive wireless power transmission technology is relatively mature, with very good transmission efficiency over short transmission distances. However, as the transmission distance increases, the transmission efficiency falls very quickly, and the relative position of the coil is the greatest challenge to the development of effective applications. 
Table 1. Three major wireless charging technology standards.

\begin{tabular}{|c|c|c|c|}
\hline & WPC & A4WP & PMA \\
\hline Logo & (j) WIRELESS POWER & $\frac{\text { Alliance for }}{\text { Wireless Power }}$ & \\
\hline Technology & $\begin{array}{c}\text { Inductive Coupling + Magnetic } \\
\text { Resonance }\end{array}$ & Magnetic Resonance & Inductive Coupling \\
\hline Main members & Philips Panasonic HTC & Samsung Qualcomm NXP & $\begin{array}{c}\text { Duracell-Powermat } \\
\text { BlackBerry NEC Starbucks }\end{array}$ \\
\hline Members & 214 & 120 & 71 \\
\hline Products & $>750$ & 0 & 10 \\
\hline Phone integrated & $>80$ & 0 & 0 \\
\hline Market & Phone/industry & Phone/tablet & Phone/tablet \\
\hline
\end{tabular}

Electromagnetic resonance refers to the use of resonant devices (inductors and capacitors) to cause the transmitter and receiver to reach a specific frequency which, in turn, generates magnetic resonance and thus energy. The advantages are high transmission efficiency and power. The disadvantage is the relatively high cost. High resonance frequency will impact transmission efficiency, but it is relatively easy to design applications for charging multiple devices simultaneously. At present, electromagnetic resonance technologies offer high efficiency, long transmission distance and strong tolerance to coil deviation.

Furthermore, $6.78 \mathrm{MHz}$ is the standard frequency for magnetic resonance technologies, and is thus used for magnetic resonance in many cases. As previously mentioned, this technology has high transmission efficiency and energy, so $6.78 \mathrm{MHz}$ wireless power transfer mostly transmits higher wattages and therefore most IC-based rectifier designs tend to use the CMOS BCD process or other processes that withstand higher voltages. The larger area brings a commensurate increase in current resistance, giving full play to the advantages of magnetic resonance technology. Commercialized designs can reach up to $1000 \mathrm{~W}$, thus another type uses Discrete Components instead of IC Rectifiers. Although this requires a larger size, it can meet requirements for voltage and current resistance, usually because it can achieve high wattage charging energy, and most products that require such high energy levels provide the necessary volume. Thus, although the IC circuits in Discrete Components are relatively large, this approach meets the requirements of applications such as recharging of electric vehicles.

In addition, $13.56 \mathrm{MHz}$ is used for magnetic induction technologies, and is thus used here for all magnetic induction technologies which are nearly all RFID applications. RFID offers advantages including low cost and the use of NFC (near-range wireless transmission) for wireless data transmission and charging over distances less than $1 \mathrm{~m}$. However, transmission power achieved using this approach is less than that of magnetic resonance, so it is mainly used for instantaneous charging and data transfer. Recently, the active development of biomedical circuits has raised the need for data transfer with low power consumption and high durability. These requirements generally overlap with RFID, and many papers on wireless energy harvesting have used $13.56 \mathrm{MHz}$. Because of the above-mentioned low transmission power, it is suitable for use in IC-based circuits, allowing for a reduction in size and current without using high voltage resistance or requiring size parameters to withstand larger currents.

Finally, the circuit design for frequency bands exceeding $433 \mathrm{MHz}$ is discussed. Compared to low frequencies, the main challenges encountered in high-frequency circuits are additional parasitic effects and matching problems. In low-frequency circuits, most of the parasitic capacitance and inductance can be regarded as infinite impedance and infinitesimal impedance. In short, most of the characteristics of low-frequency circuits serve to 
maintain the characteristics of transistors and circuits, and do not entail excessive parasitic effects to affect circuit performance. However, in the high-frequency band, the original parasitic capacitance and parasitic inductance are caused by the high-frequency effect, resulting in a prominent parasitic effect. A common problem is the matching of the input impedance at the two ends of the differential input. For the design of a differential pair, if the input at both ends is excessively severe because of the mismatch effect, the input voltage at both ends will not be a differential input, causing problems for the back-end circuit operation. Therefore, the designer must conduct more accurate simulations and calculations of the inductance and capacitance of the input end.

\section{AC-DC Conversion Circuit Architecture}

In Figure 2, we have seen where the Power Recovery Module is located. It serves as an AC-DC conversion system for the implanted chips. The rectifier inside is used to receive the wireless carrier transmitted through the skin. It belongs to AC-DC and plays a core role. Its corresponding input terminal is the receiving antenna inside the body (usually close to the skin). Through the concept of resonance, the induced electromotive force is generated and then induced in the body. The induced current at the receiving end forms the voltage on the receiving chip through the load, and supplies power to the implanted SOC in the body. However, because the power supply is essentially an AC signal, it needs to be rectified by a rectifier circuit, and the output waveform is an imperfect DC signal needing to be further filtered by a low-pass filter for high-frequency removal. In order to ensure that the power supply of the SOC in the body can remain stable even under the condition of receiving voltage fluctuations, it is necessary to have it passed a low-pass filter.

The downstream voltage regulator circuit is used to regulate the output of the filter, so that the power supply to the implanted SOC in the body can reach a sufficiently low ripple to ensure that the SOC in the body will not malfunction and cause physiological damage. The received voltage fluctuation is mainly due to the distance variation between the transmitter and the receiver. Owing to the nature of the system setups in hardware, their relative distance cannot be kept absolutely fixed, resulting in a change in the coupling coefficient of the two coils. Poor coupling coefficient compromises the conversion efficiency due to the increase in the distance between the transmitter in vitro and the receiver in the body.

The efficiency decayed exponentially, aggravating the difficulty of the internal circuit design of the AC-DC in the body. The rectifier itself is responsible for different forms of energy conversion, and its important performance index is the conversion efficiency, which is mainly divided into voltage conversion efficiency VCE and power conversion efficiency PCE. VCE is defined as ratio of the output voltage (numerator) to input voltage (denominator) of the rectifier circuit (expressed as percentage), while PCE is defined as the ratio of the output power (numerator) to the input power (denominator) of the rectifier circuit (also expressed as a percentage). The higher the value of the two, the better the designed rectifier. Efficiency usually comes with the trade-off of the design complexity.

\subsection{Full-Wave Diode Bridge Rectifier}

Conventional rectifiers use a PN interface for a Full-wave Bridge Rectifier configured using four PN diodes or a Schlocky Barrier Diode to change the current flow. Because the diodes' reverse bias characteristic, there is no leakage current, thus minimizing unnecessary energy loss, but the output voltage will be depleted by the diode cross-pressure $\left(\mathrm{V}_{\text {th }}\right)$. The relatively low voltage conversion rate results in a low overall conversion rate. Figure $3 a$ shows the circuit, while Figure $3 b$ shows a Full-wave Bridge Rectifier simulated in a $180 \mathrm{~nm}$ standard CMOS single-poly and six-metal process, including 3.3-V tolerable I/O devices. The circuit design is relatively simple and stable. However, this rectifier consumes twice the diode's threshold voltage $\left(\mathrm{V}_{\text {th }}\right)$, with the maximum output voltage calculated by Equation (1):

$$
\mathrm{V}_{\text {out,MAX }}=\mathrm{V}_{\text {in }}-2 \mathrm{~V}_{\text {th }}
$$


where the diode forward bias voltage $\mathrm{V}_{\text {th }}$ is $700 \mathrm{mV}$, and the Schottky diode has a threshold voltage of about $300 \mathrm{mV}$. Therefore, applying the diode bridge rectifier causes an excessively large voltage drop in low-voltage implanted devices, resulting in significant reductions in voltage conversion efficiency (VCE) and power conversion efficiency (PCE). These diodes thus cannot be used in the integrated circuit, thus increasing the implant size. As chip design and manufacturing capabilities improve, the resulting implant device circuits will require less power to operate. Using a diode bridge rectifier for low-power requirements will increase the ratio of wasted voltage, resulting in the PCE and VCE having a greater influence.

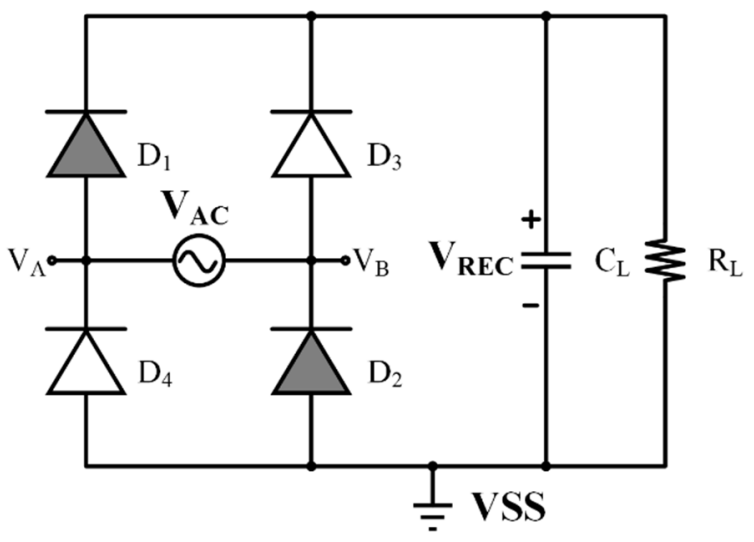

(a)

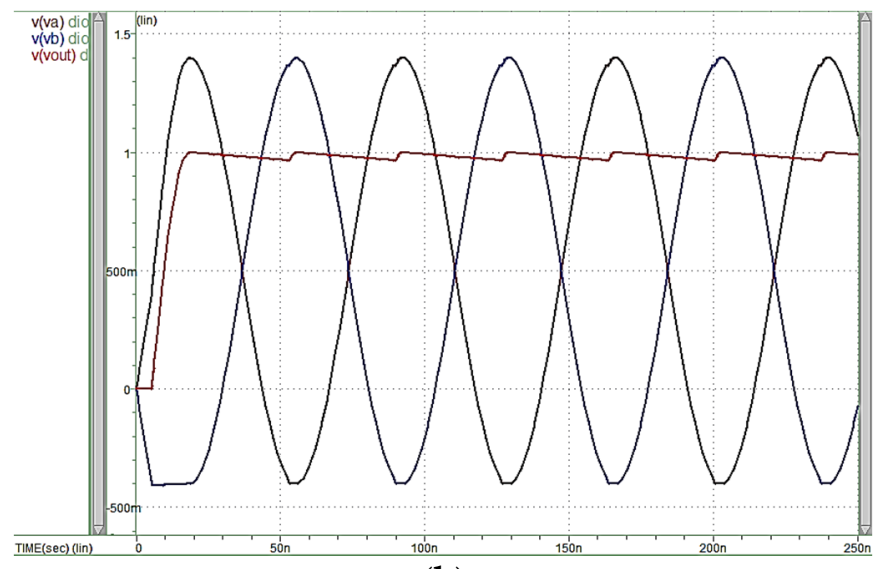

(b)

Figure 3. (a) Full-wave diode bridge rectifier architecture. (b) Simulated full-wave diode bridge rectifier $\left(\mathrm{V}_{\text {th }} \approx 0.4 \mathrm{~V}\right)$.

\subsection{Dual Cross-Coupled Rectifier}

Figure 4a shows the above-mentioned Dual Cross-Coupled Rectifier, which presents an advantage in that it uses negative-resistance architecture to load the pull-up and pulldown paths, thus completely eliminating the pressure drop caused by the rectifier on the diode. The negative resistance cross-coupled circuit acts as a switch which is open if only a very small channel resistance is selected, thus greatly reducing the voltage drop. The circuit operates as follows: When the $V_{A}$ is high and $V_{B}$ is low, $M_{N 2}$ and $M_{P 1}$ are on. At this time, $V_{B}$ will connect to the ground, and $V_{A}$ will charge the capacitor. Otherwise, when $V_{B}$ is high and $V_{A}$ is low, $M_{N 1}$ and $M_{P 2}$ are open, $V_{A}$ is grounded and $V_{B}$ charges the capacitor. However, after $V_{B}$ reaches its peak voltage, $V_{B}$ is still higher than $V_{A}$ despite the falling input voltage. At this time, the switch is not closed, and the charging path is still present. At this time, if $V_{B}$ is lower than $V_{R E C}$, this path becomes the path of discharge. Therefore, the Dual Cross-Coupled Rectifier will continue to charge and discharge the capacitor. The Dual Cross-Coupled Rectifier overcomes the dual diode voltage drop of the bridge rectifier, thus improving power conversion efficiency. However, the constant charging and discharging of the reverse current will result in significant power consumption by the rectifier. Figure $4 \mathrm{~b}$ shows a simulation of the Dual Cross-Coupled Rectifier, indicating that it can overcome the diode's voltage drop, but will produce a reverse current. 


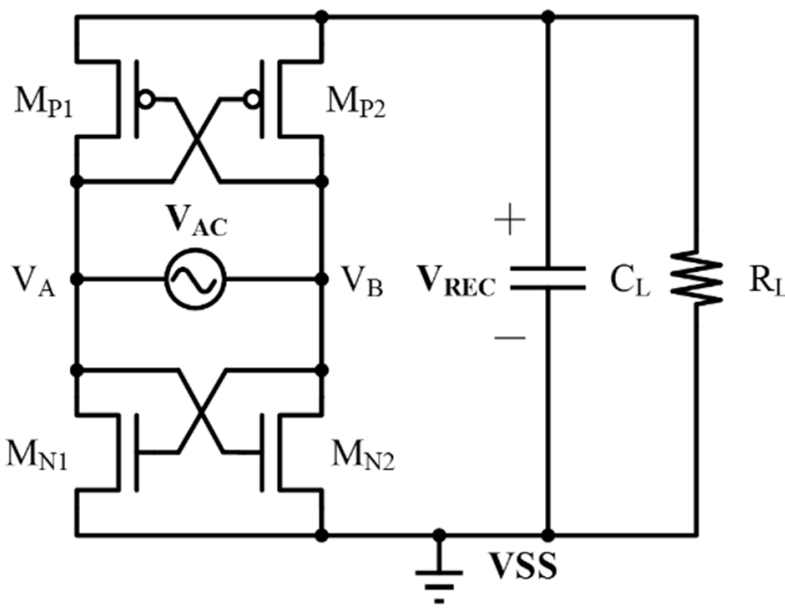

(a)

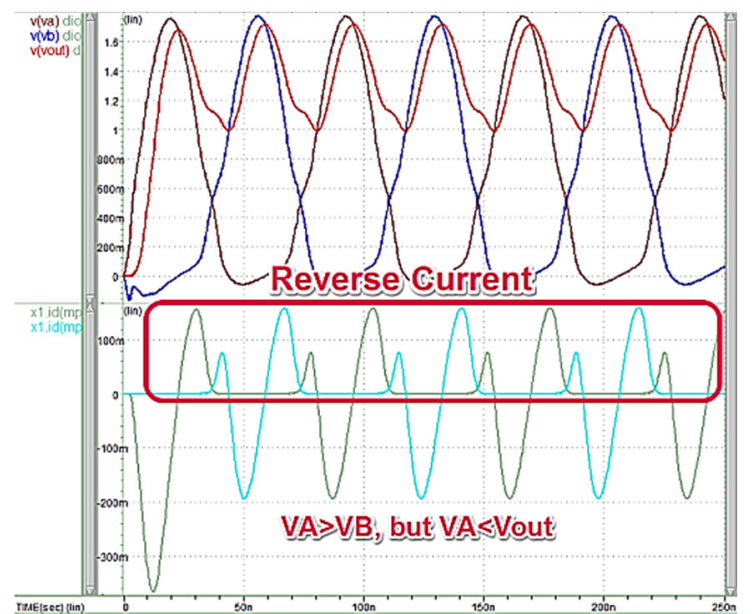

(b)

Figure 4. (a) Dual Cross-Coupled Rectifier Architecture. (b) Simulated Dual Cross-Coupled Rectifier $\left(\mathrm{V}_{\text {th }} \approx 0.4 \mathrm{~V}\right)$.

\subsection{Hybrid Rectifier}

In addition to the above-mentioned simple structures, a hybrid rectifier, as shown in Figure 5a, provides a set of diodes to prevent current flow [5]. The conduction path only requires a single diode to prevent a countercurrent, thus $\mathrm{M}_{\mathrm{P} 1}$ and $\mathrm{M}_{\mathrm{P} 2}$ can, respectively, be used as the positive and negative half-cycle anti-countercurrent diodes. Once the circuit achieves anti-countercurrent function, the lower half exhibits essentially no drop in voltage consumption in the negative resistance cross-coupled circuit (i.e., $\mathrm{M}_{\mathrm{N} 1}$ and $\mathrm{M}_{\mathrm{N} 2}$ ). To effectively prevent the Latch-up effect (parasitic vertical PNP transistors), and to reduce substrate leakage, the PMOS body must be kept at the highest level, thus requiring auxiliary transistors $\mathrm{M}_{\mathrm{P} 3} \sim \mathrm{M}_{\mathrm{P} 6}$. In terms of left/right symmetry, we take the right half as an example. When the node $V_{A}$ rises to the high level, $M_{N 1}$ will first use VSS to connect to VB. Next, $M_{P 1}$ will turn on to form a loop to cause capacitance charging between $V_{\text {REC }}$ and VSS. Because the gate and transistor source for $\mathrm{M}_{\mathrm{P} 1}$ and $\mathrm{M}_{\mathrm{P} 3}$ are linked together, when $\mathrm{M}_{\mathrm{P} 1}$ is turned on, $\mathrm{M}_{\mathrm{P} 3}$ will also turn on. The $\mathrm{V}_{\mathrm{A}}$ potential will be received by the $\mathrm{M}_{\mathrm{P} 3}$ drain/body, causing the $\mathrm{N}$-Well to remain close to the maximum potential (i.e., the maximum potential minus a $\mathrm{P}-\mathrm{N}$ Junction conduction voltage). When the $\mathrm{V}_{\mathrm{A}}$ voltage drops below $\mathrm{V}_{\mathrm{REC}}+|\mathrm{Vtp}|$ hours (where $|\mathrm{Vtp}|$ is the absolute value of the PMOS threshold voltage, which here is $\mathrm{M}_{\mathrm{P} 1}$ ), then $M_{P 1}$ and $M_{P 3}$ will both be OFF. When $V_{A}$ falls $|V t p|$ below $V_{R E C}, M_{P 4}$ will be on, and the body will receive $V_{\text {REC }}$ to ensure the body is at the highest potential, i.e., that of the source. The hybrid architecture combines the advantages of the bridge rectifier and the cross-coupled rectifier, incurring a smaller diode voltage drop than the bridge rectifier, thus improving power conversion efficiency. It also incurs less countercurrent than the cross-coupled rectifier, resulting in further power savings. Simulation results are shown in Figure $5 b$.

\subsection{CMOS Active Rectifier Using Pull-Up $V_{\text {th }}$ Elimination Technology}

Active rectifiers using pull-up technology [6] use the $\mathrm{M}_{1}$ to $\mathrm{M}_{4}$ modules to construct key rectified transistors with upstream $\mathrm{M}_{1,2}$ using PMOS, and downstream $\mathrm{M}_{3,4}$ using NMOS. When $V_{\text {in }}>V_{\text {out }}$, it provides a current path from the input to the load terminals and uses the pull-up control circuit to integrate $\mathrm{M}_{5}-\mathrm{M}_{8}$ for the $\mathrm{M}_{9,10}$ control gate voltage, with the circuit shown in Figure 6a. $M_{9,10}$ causes the $M_{3,4}$ gate voltage to ground. When $\mathrm{V}_{\mathrm{in}}<\mathrm{V}_{\text {out}}$, the control $\mathrm{M}_{3,4}$ is in the cutoff area, and $\mathrm{M}_{1,2}$ and $\mathrm{M}_{3,4}$ simultaneously turn off the path from the charged load capacitor's reflow current to the input terminal. 


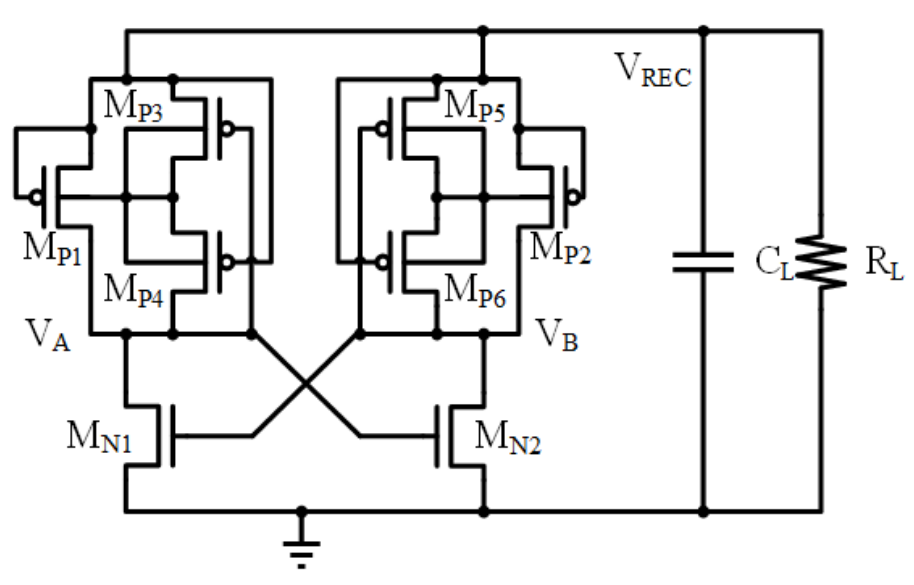

(a)

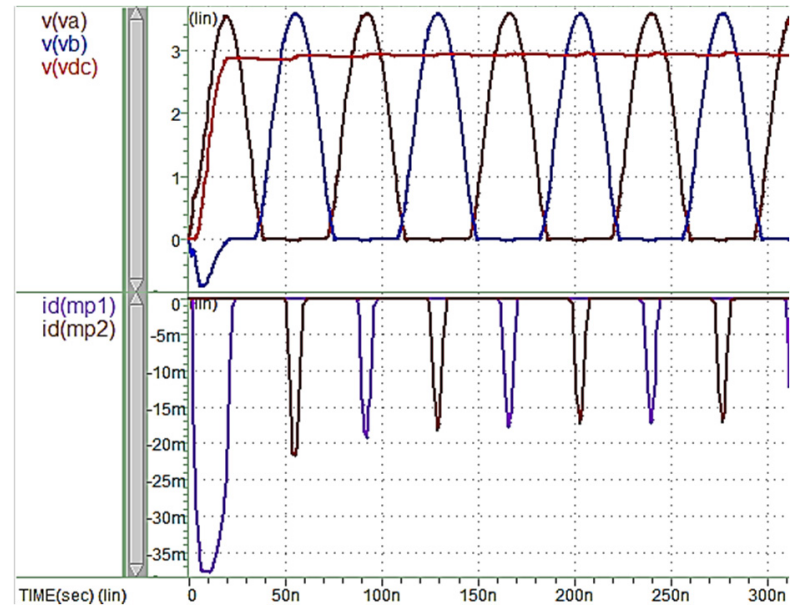

(b)

Figure 5. (a) Hybrid Rectifier Architecture. (b) Simulated Hybrid Rectifier.

During the positive period, $\mathrm{M}_{1}$ turns on and then activates the connection between the output and input terminals such that the signal source which ignores the possible resistance drop is set to the grounded voltage relative to $V_{\text {out }}-V_{\text {in }}$. If the input terminal pressure $V_{\text {in }}>V_{\text {out }}$, the input terminal voltage is lower than the ground. Thus, when the input voltage is lower than the ground for one $\mathrm{V}_{\mathrm{th}}, \mathrm{M}_{7,8}$ is turned on, conducting low voltage to the $\mathrm{M}_{5,6}$ gate. This forces $\mathrm{M}_{5,6}$ to open, and also pull up $\mathrm{M}_{4}$ to the open state. Figure $6 \mathrm{~b}$ shows the partial circuit of the positive half-wave cycle. The simulated pull-up for the active rectifier output results are shown in Figure $6 c$, while the state of each transistor in each half-wave cycle is shown in Figure $6 \mathrm{~d}$. When $V_{\text {in }}<V_{\text {out }}, M_{7,8}$ is off, and the pull-up PMOS transistor is used to connect the $\mathrm{M}_{5,6}$ gate to the $\mathrm{V}_{\text {out }}$, and the $\mathrm{M}_{3,4}$ transistor gate is driven by the same voltage. In the negative half-cycle, the same method is used, and these two rectifier circuits are used to achieve rectification. A frequency of $10 \mathrm{MHz}$ and an input voltage Vp-p of $2.2 \mathrm{~V}$ achieves the best PCE of $79 \%$, and the relationship between measured power conversion efficiency and input voltage scan is shown in Figure 6e.

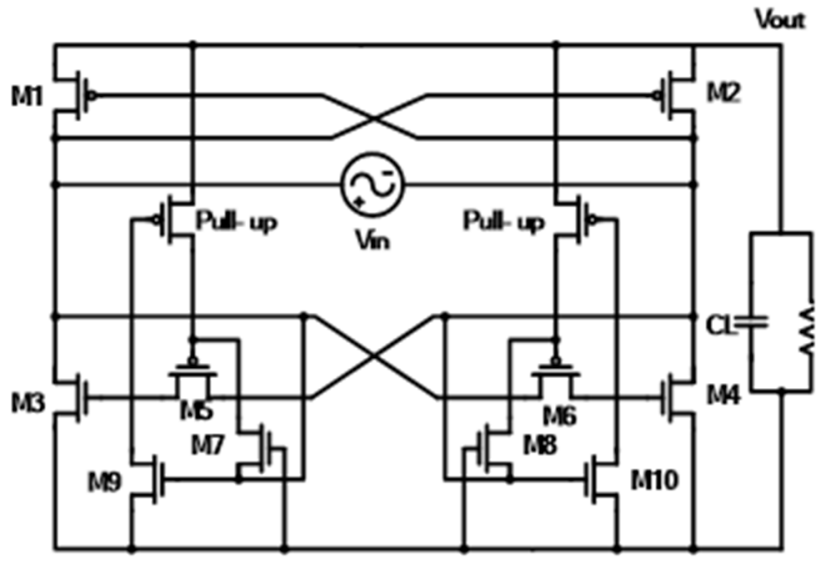

(a)

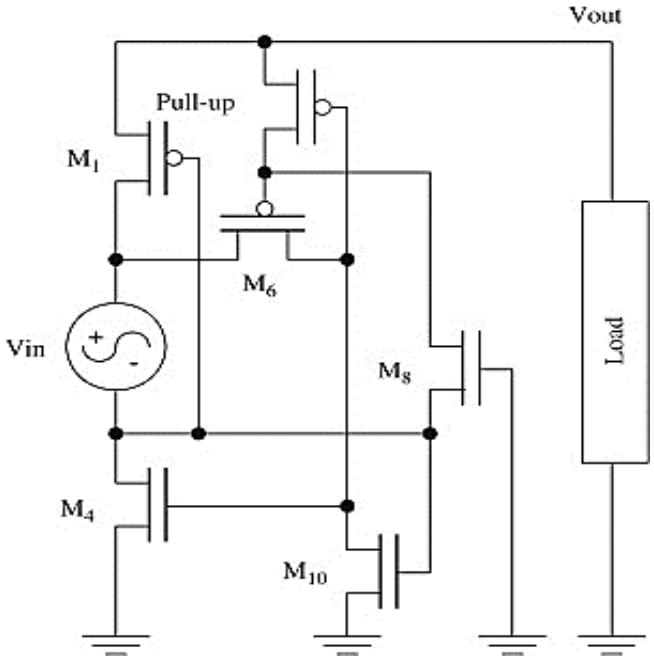

(b)

Figure 6. Cont. 


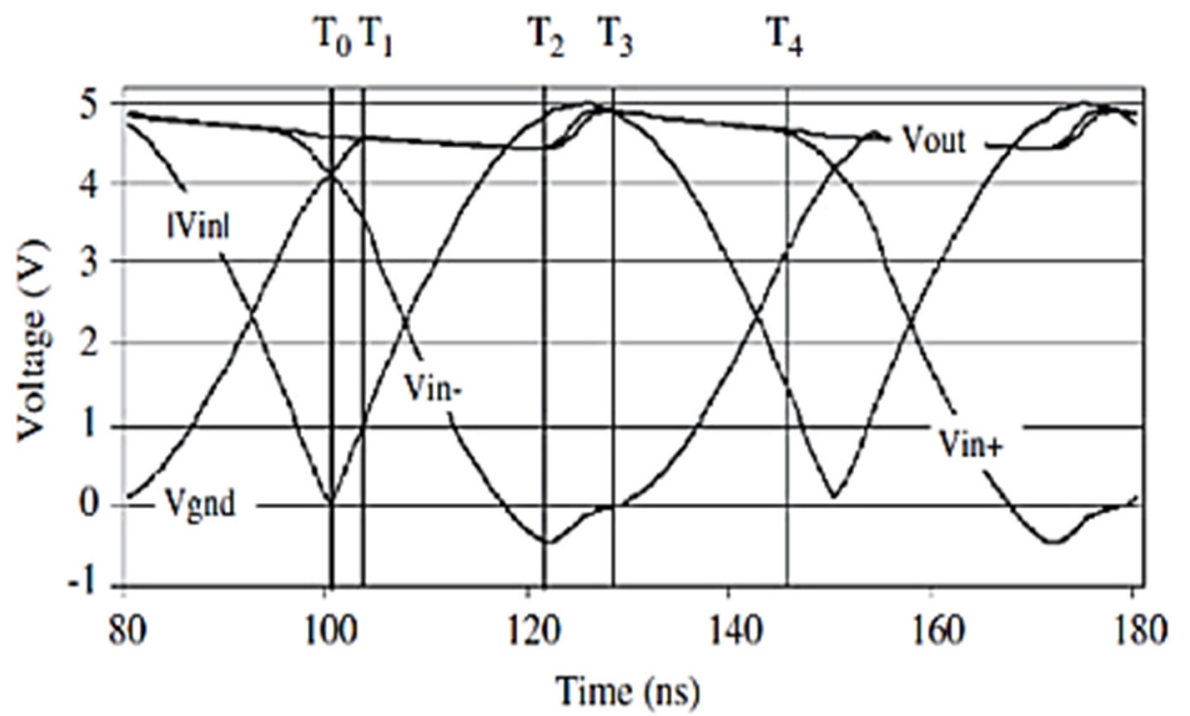

(c)

Rectifier switching states for positive cycle.

\begin{tabular}{llllll} 
& $M_{1}$ & $M_{4}$ & $M_{6}$ & $M_{8}$ & $M_{10}$ \\
\hline$S_{0}$ & OFF & OFF & OFF & OFF & OFF \\
$S_{1}$ & ON & OFF & OFF & OFF & ON \\
$S_{2}$ & ON & ON & ON & ON & OFF \\
$S_{3}$ & ON & OFF & OFF & OFF & ON \\
$S_{4}$ & OFF & OFF & OFF & OFF & ON \\
\hline
\end{tabular}

(d)

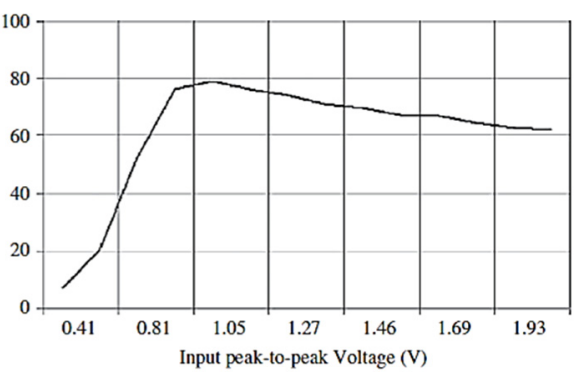

(e)

Figure 6. (a) Active rectifier using pull-up technology [6]. (b) Schematic diagram of the half-wave cycle for each transistor initialization [6]. (c) Simulated pull-up active rectifier output results [6]. (d) Rectifier switching states for positive cycle [6]. (e) power conversion efficiency and input Voltage scan [6]. Reprinted with permission from Ref. [6]. Copyright (C) 2009 Elsevier Ltd.

\subsection{CMOS Active Rectifier with Resistance Division $V_{\text {th }}$ Elimination Technology}

This rectifier uses a threshold voltage cancellation technique to reduce the voltage drop at the conduction threshold [7], using two PMOSs for the $\mathrm{M}_{5}$ and $\mathrm{M}_{6}$ upstream of the rectifier, and using a cross-coupled bias to reduce $\mathrm{M}_{5}$ and $\mathrm{M}_{6}$ conduction when the threshold voltage is affected. The other part uses two NMOSs as upstream rectifiers for $\mathrm{M}_{3}$ and $\mathrm{M}_{4}$, but the $\mathrm{M}_{3}$ and $\mathrm{M}_{4}$ transistor gate bias voltage is connected with the bias obtained by the $\mathrm{M}_{1}$ and $\mathrm{M}_{2}$ transistors. This dynamic bias voltage is lower than the threshold voltage of $\mathrm{M}_{3}$ and $\mathrm{M}_{4}$ and its average voltage can be adjusted according to the size of $\mathrm{M}_{1}$ and $\mathrm{M}_{2}$ along with the resistance values $R_{1}$ and $R_{2}$. When one of the $M_{3}$ or $M_{4}$ transistors is turned on and the other is off, the high bias voltage will be less than $R_{1}$ and $R_{2}$ during the turn-on period. However, the actual bias voltage is too close to the transistor's threshold voltage, causing current leakage from the output terminal's $\mathrm{C}_{\mathrm{L}}$ to the input terminal. Therefore, completely eliminating the threshold voltage bias value will reduce the PCE. Thus, 70\% of the $\mathrm{M}_{3}$ and $\mathrm{M}_{4}$ are conductively and non-conductively connected to the cross-coupled resistor to store the voltage to achieve a better tolerance range between $65 \%$ and $75 \%$. The transistors $\mathrm{M}_{3}$ and $\mathrm{M}_{4}$ conduct when the negative RF signal is $150 \mathrm{mV}$ lower than $\mathrm{V}_{\mathrm{REC}}$. The simulated analog waveform where, following distribution, the $\mathrm{R}_{\mathrm{L}}$ for the rectifier is $10 \mathrm{k} \Omega$ and $\mathrm{C}_{\mathrm{L}}=1 \mathrm{nF}$. This technology achieves a PCE of $85.8 \%$ at a frequency of $40.69 \mathrm{MHz}$ and a load resistance of $8 \mathrm{~K} \Omega$. 


\subsection{Bootstrapped Rectifier}

A bootstrapped rectifier circuit is presented in [8]. The cross-coupled MOS structure is still used as negative resistance, operating like a switch without bipolar pressure drop issues, while the upper half is used like a belt circuit. Because the NMOS has better drive capability than PMOS, it can achieve the same current drive with a smaller size. However, the NMOS can only achieve a maximum charge equal to the maximum source charge below one $V_{\text {th }}$ (where $V_{\text {th }}$ is the threshold voltage of the NMOS transistor). Therefore, we use a bootstrap rectifier to raise the NMOS gate voltage one $V_{\text {th }}$ above that of the maximum input voltage, and we allow NMOS to act like a PMOS switch to achieve the maximum potential for the general output terminal. The right half of the circuit diagram is the bootstrap circuit, where the circuit principals operate as follows. When $V_{A}$ is high and $V_{B}$ is low, $M_{N 1}, M_{N 2}$ and $\mathrm{M}_{\mathrm{N} 3}$ conduct, while $\mathrm{M}_{\mathrm{P} 1}, \mathrm{M}_{\mathrm{N} 4}$ and $\mathrm{M}_{\mathrm{N} 5}$ are closed. At this time, the capacitor $\mathrm{CB}$ is charged by $\mathrm{V}_{\mathrm{A}}$. When the high and low states swap, $\mathrm{M}_{\mathrm{N} 1}, \mathrm{M}_{\mathrm{N} 2}$ and $\mathrm{M}_{\mathrm{N} 3}$ are closed, while $\mathrm{M}_{\mathrm{P} 1}, \mathrm{M}_{\mathrm{N} 4}$ and $\mathrm{M}_{\mathrm{N} 5}$ are open. The $\mathrm{M}_{\mathrm{N} 5}$ gate voltage will exceed the maximum input voltage such that VRectifier can reach VC2 with virtually no pressure drop, thus completely resolving the diode voltage waste produced by the bridge rectifier. Although this rectifier overcomes the problem of the bipolar voltage drop, it still poses a reverse current problem with the cross-coupled rectifier, resulting in power loss and reduced conversion efficiency.

\subsection{Full-Wave Active Rectifier}

Comparing the above architectures, we find that the bridge rectifier achieves the goal of reducing the diode voltage drop, but later structures are unable to prevent the inevitable countercurrent problem. However, charging and discharging occurs with every half wave cycle, thus increasing the power consumption above the threshold voltage of the bridge rectifier. Therefore, resolving the charge-discharge problem presents a choice between diode voltage drop and countercurrent, raising the need for an optimal method that uses at least one diode to open a conduction path because the diode itself is one of the most natural countercurrent preventions, though it still incurs a diode voltage drop. Although commercially available Schottky diodes can be used to achieve high conversion efficiency in the rectifier, their integration poses problems in terms of component size, thus limiting their applicability. A review of the architecture reveals that both diode connection and negative resistance cross-coupled connection structures act as switches, where the only difference is that the diode must conduct the threshold voltage. That is, when one terminal of the receiving coil is below the output voltage by a threshold voltage or more, the rectifier shuts off, thus limiting the maximum chargeable level of the rectifier output. The negative resistance connection is not limited by this threshold voltage. However, when the rectifier output exceeds any input, the charge stored by the rectifier output load when the other input is still low will discharge along the original charging path, resulting in reverse leakage.

\subsection{Active Diode Rectifier}

The circuit structure proposed in [9] achieves high conversion efficiency for the rectifier circuit without a voltage limit or countercurrent, which is equivalent to having a rectifier with two switch circuits (see Switch 0 and Switch 1). Assuming that VCOILa is in a higher state than VCOILb, if VCOILa is higher than Vrecout, then Switch 0 is turned on, causing the current to flow back to VCOILb through the path Switch $0->C_{L} \rightarrow N_{2}$, thus charging $\mathrm{C}_{\mathrm{L}}$. However, if Vrecout is higher than VCOILa, Switch 0 may close the return path, thus completely eliminating the threshold voltage and current flow problems. Similarly, when VCOILb is higher than VCOILa, if VCOILb is higher than Vrecout, Switch 1 will turn on, causing the current to flow back to VCOILa through the path Switch $1 \rightarrow \mathrm{C}_{\mathrm{L}} \rightarrow \mathrm{N}_{1}$. However, when Vrecout is higher than VCOILb, Switch 1 will close. This concept is referred to as an active diode rectifier architecture.

The authors in [9] proposed a special rectifier architecture, where one side of the rectifier (VCOILb versus Vrecout) is the same as the other side (VCOILa versus Vrecout). 
Here, only one side of the circuit structure is explained. The structure of this circuit is similar to most previously described rectifiers in that it consists of two conduction paths, one of which consists of the transistors $\mathrm{M}_{\mathrm{P} 1}$ and $\mathrm{M}_{\mathrm{N} 1}$, and these two conduction paths, respectively, pass through the positive and negative half-cycle conductors. Transistors $\mathrm{M}_{7}$ and $\mathrm{M}_{8}$ are used to keep the PMOS body at a high potential to prevent the latching effect. The $\mathrm{M}_{1}-\mathrm{M}_{6}$ and $\mathrm{M}_{\mathrm{R} 1}-\mathrm{M}_{\mathrm{R} 6}$ transistors serve as a comparator circuit. When VCOILa is higher than Vrecout, it can cause $\mathrm{M}_{\mathrm{P} 1}$ to completely open, and vice versa. To ensure sufficient voltage and power conversion efficiency for the entire rectifier circuit (i.e., to minimize loss), the $\mathrm{M}_{\mathrm{R} 1}-\mathrm{M}_{\mathrm{R} 6}$ transistors should be as large as possible to operate with large resistance. Let Vrecout be $0 \mathrm{~V}$.

The current flowing through $\mathrm{M}_{4}$ is very small, and the gate voltage (or $\mathrm{M}_{1}$ ) can be estimated to be about Vrecout - $\left|V_{\text {th } 4}\right|$ (where $\left|V_{\text {th4 }}\right|$ is the absolute voltage of $M_{4}$. Therefore, $\mathrm{M}_{1}$ will turn on when the gate voltage of $\mathrm{M}_{1}$ is less than VCOIL, which is greater than the absolute value of the limiting voltage $\left|V_{\text {th } 1}\right|$ (i.e., VCOILa $>$ Vrecout $-\left|V_{\text {th } 4}\right|$ $\left.+\left|V_{\text {th } 1}\right|\right)$. The formula VCOILa $>$ Vrecout $-\left|V_{\text {th1 }}\right|$ shows that if $\left|V_{\text {th } 4}\right|$ and $\left|V_{\text {th } 1}\right|$ are equal, then $\mathrm{M}_{1}$ will turn on when VCOILa $>$ Vrecout. This causes the $\mathrm{M}_{6}$ gate voltage to pull-up, causing $\mathrm{M}_{6}$ to open and $\mathrm{M}_{5}$ to close, while $\mathrm{M}_{2}$ is also closed and $\mathrm{M}_{\mathrm{P} 1}$ is turns on. On the other hand, $M_{2}$ will open when Vrecout $>$ VCOILa $-\left|V_{\text {th } 3}\right|+\left|V_{\text {th2 }}\right|$. When $M_{2}$ turns on, the gate voltage of $\mathrm{M}_{\mathrm{P} 1}$ is pulled up by Vrecout to a level sufficient to turn off $\mathrm{M}_{\mathrm{P} 1}$, thus eliminating the current. Here, if $\mathrm{M}_{2}$ is designed with a sufficiently large current driving force, the $M_{P 1}$ gate voltage will be pulled up to close to the $M_{P 1}$ level, thus almost completely eliminating the countercurrent. However, this will result in the loss of the charge stored in the Vrecout, thus reducing conversion efficiency. Therefore, the design requires careful consideration. Despite these advantages, the conduction path of this architecture includes a diode $\mathrm{M}_{\mathrm{N} 1}$. Thus, when the entire charging path is activated, the charge from Vrecout to the ground will be less than VCOILa - VCOILb by one $\mathrm{M}_{\mathrm{N} 1}$ threshold voltage $\left|\mathrm{V}_{\mathrm{thN} 1}\right|$. Nonetheless, since $\mathrm{M}_{1}-\mathrm{M}_{6}$ and $\mathrm{M}_{\mathrm{R} 1}-\mathrm{M}_{\mathrm{R} 6}$ have been implemented as a comparator circuit, the countercurrent can be effectively clamped.

\subsection{Two-Stage Active Rectifier}

A two-stage active rectifier in [10] can be divided into two levels. The first level is a Negative Voltage Converter (NVC), and the second level is an active diode. In the first level, the NVC circuit output point A is connected to the active rectifier input in the second level. Point $B$ is connected to the ground, and the NVC primarily serves to ground the input from the $V_{\text {in }}$ positive signal through $M_{N 1}$ and $M_{N 2}$. When $V_{i n 1}>V_{i n 2}, M_{N 2}$ is turned on and $V_{\mathrm{in} 2}$ is grounded, thus $M_{P 1}$ is turned on and $V_{\mathrm{in} 1}$ is grounded at point $A$. Conversely, when $V_{\text {in2 }}>V_{\text {in1 }}, M_{N 1}$ is turned on and $V_{i n 1}$ is grounded, thus $M_{P 2}$ is turned on and $V_{\text {in2 }}$ is grounded at point $\mathrm{A}$. Therefore, the NVC circuit can convert the positive and negative half-cycles to a half-cycle signal that is positive compared to the GND. The benefit of this is that only terminal A requires an active diode, but the cost will be reflected in the operating frequency of the active diode. When the positive and negative half-cycles use the same diode, the comparator's operating frequency will double. In other words, the comparator's bandwidth and speed must be increased, while the power consumption of the active diode MPS is not reduced.

\subsection{Highly Efficient Active Rectifier}

For the above-mentioned active full-wave rectifier, the authors in [9] propose a comparator-based active diode, but the conversion efficiency of this approach is limited by the ability of the gate voltage to drive the switch. Thus, a driving buffer is needed to improve the slew rate. A similar problem is frequently found in DC-DC power conversion circuits. On the other hand, the authors in [10] use a total of six large transistors, of which four are used for half-wave conversion, and one MPBD is used as a passive diode, and MPS is the active diode. This results in reduced size efficiency. In contrast with RFID specifications, active rectifiers typically operate at a frequency of $13.56 \mathrm{MHz}$. In other words, 
the comparator operating frequency should be higher than this, but the diode proposed in [10] requires an operating frequency of $27.12 \mathrm{MHz}$ for normal operation. In view of this, a two-stage high-frequency rectifier is unsuitable, and the authors in [11] provide a better driving force for active diodes. In this approach, the use of a comparator and driving buffer improve the delay caused by the countercurrent.

This active rectifier uses four key conduction current flow: two PMOS and two NMOS transistors, respectively called $\mathrm{P}_{1,2}$ and $\mathrm{N}_{1,2}$. In [11], the external control CTL [0:3] compensates for the improved comparator. To drive the larger $\mathrm{P}_{1,2}$ transistor at a high frequency of $13.56 \mathrm{MHz}$, one typically adds a buffer after the comparator as a driver. The comparator's operating speed is limited by a time delay (TP) to determine the output response speed as the input speed changes. Due to the TP-High-to-Low (TP-HL) delay, the comparator is not in time to turn on $\mathrm{P}_{1,2}$ and reduce the power transmission. This delay is transmitted to the load. In addition, the TP-Low-to-High (TP-LH) delay causes the comparator to delay the closing of $\mathrm{P}_{1,2}$. These delays cause the PCE to fall when $\mathrm{V}_{\text {in }}$ $<V_{R E C}$, and the charged current is returned from the $C_{L}$ to the secondary side coil. The TP cannot fall to zero, thus a common gate comparator (CG CMP) is used and two offset control modules (OffsetF and OffsetR) and a current shortage (CS) inverter are provided. The input of the CG comparator that injects the programmable deviation current OS-F and OS-R is dependent on the feedback status signals FB-F and FB-R of the $\mathrm{V}_{\text {out }}$. Thus, $\mathrm{V}_{\text {out }}$ time can be used to perceive rising and falling signals in advance.

With regard to the sensing timing of FB-F and FB-R, when $V_{\text {in } 1}-V_{\text {in2 }}>V_{R E C}, V_{\text {out }}$ decreases. The CG comparator outputs the $V_{\text {out }}$ low voltage to open $P_{1,2}$ and causes the $V_{\text {in }}$ current to charge to $V_{\text {REC }}$. At this point, the FB-F signal is low, and only the OffsetF module injects current into the CG comparator. Thus, before the $V_{\text {in1 }}-V_{\text {in2 }}$ voltage reaches $V_{R E C}$, $\mathrm{V}_{\text {out }}$ is forced to fall. When $\mathrm{V}_{\text {in } 1}-\mathrm{V}_{\mathrm{in} 2}<\mathrm{V}_{\mathrm{REC}}$, FB-R falls, followed by $\mathrm{V}_{\text {out }}$, opening the offset block to inject the current into the $C G$ comparator, thus forcing $V_{\text {out }}$ to rise when $V_{\text {in1 }}-V_{\text {in2 }}$ is lower than $V_{R E C}$. However, this incurs some serious side effects. If OffsetR turns on after $V_{\text {out }}$ begins to fall, this instantaneous negative feedback mechanism limits $\mathrm{V}_{\text {out }}$ from falling completely and affects $\mathrm{V}_{\text {out }}$ jitter. The experimental results simulating a $\mathrm{C}_{\mathrm{L}}$ of $10 \mathrm{UF}$ and a frequency measure of $13.56 \mathrm{MHz}$ produces a maximum PCE of $84.5 \%$. The size of each transistor and offset comparator rectifier is optimized for operation at 13.56 MHz. However, PCE decreases due to the comparator delay at higher frequencies. At lower frequencies, the PCE also drops slightly, turning off the rectifier earlier due to a fixed comparison offset.

The authors in [11] provide a better switch-driving force and suggest that the offset current can be injected to control the judgment level of the comparator to achieve an early decision by the comparator and thus reduce the countercurrent. Therefore, in recent years, more studies have focused on improving effective control of the comparator to allow the entire system to automatically compensate and nearly eliminate the countercurrent. Additionally, improving switching accuracy will improve the power conversion rate (PCE).

\subsection{Adaptive Delay-Compensated Active Rectifier}

The authors in [12] implement a mechanism for automatic compensation. The authors in [12] show the active diode circuit architecture, including a push-pull common-gate comparator, a switching delay compensation circuit, a drive circuit and a power transistor. The push-pull common-gate comparator has a high slew rate and response speed and has been increasingly used in research over the past few years. This study adopts the same method used in [11] to adjust the delay by injecting current into the comparator.

The compensation method uses the gate switch signal VGN1 to obtain a sample when the switch is turned on for the difference between the inputted Vac1 voltage and the ideal voltage. This voltage difference is used to bias the source of the input current. Switching on and off causes different delays and raises the need for using two paths to obtain independent control of the on/off offset. This design provides more accurate delay compensation for independent determination and control. 
In the high-performance rectifier, the timing of turning on the active diode is important. Thus, sensing and adjusting the power transistor gate (VGN1) signal is the most basic method of correcting the delay. The delay compensation circuit is not turned on until after about 30 us, and Iac1 shows that the active diode generates a large countercurrent due to the delay, resulting in a relatively low voltage conversion rate (VCE) and power conversion efficiency (PCE).

The converted output voltage VDC is also low. When the delay compensation circuit is turned on, it tends to lock at about 100 us. The results show that the active rectifier has no inverse current, and the output voltage VDC is also higher, with a higher VCE and PCE. The PCE measurements, showing a significant increase in both the load change and input and output voltage changes after dynamic compensation is activated, indicate the importance of precision compensation.

There have recently been more works presented in the literature [13-19]. Despite the different design philosophies, the underlying purposes are similar. We compare all of them (Table 2) to give the readers a comprehensive idea about what achievements have been made for the applications. More perspectives on the application of MEMS to implantable microelectronics such as retinal chips can be found in $[20,21]$.

In today's biomedical energy-harvesting applications, as mentioned in the previous section, the power source strength may not be always stable. From the research results of reference [22], we know that one can use a time-multiplexing mechanism to perform energy harvesting and achieve maximum power point tracking (MPPT). References [23-25] mention different ways to improve the energy-harvesting systems.

In [23], the authors mentioned various issues and tradeoffs in design, as well as the definition of MPPT along with how to measure it. In terms of biomedical energy harvesting, to achieve the same concept of MPP, it is necessary to perform impedance matching under the premise of the target back-end load and the given limited input, to achieve the maximum rectifier power output.

In [24], the design optimization technology is especially introduced for the application of several micro-scale energy-harvesting systems. The application and circuit technology of the multiple-stage rectification and multiple-input multiple-output energy harvesting are introduced.

In [25], an emerging as well as promising technology known as a supercapacitor is discussed, along with how to achieve MPPT based on energy stored in supercapacitors through circuit technology. Although solar-powered energy harvesting is its applied object, the study still has considerable technical reference value. Although the studied points in these papers are not the same as in this review paper, they can enrich the content and depth.

Table 2. Comparison of recent prior articles.

\begin{tabular}{cccccccc}
\hline Reference & [13] & [14] & [15] & [16] & [17] & [18] & [19] \\
\hline Year & 2016 & 2015 & 2016 & 2014 & 2016 & 2017 & 2018 \\
\hline Frequency & $6.78 \mathrm{MHz}$ & $6.78 \mathrm{MHz}$ & $6.78 \mathrm{MHz}$ & $13.56 \mathrm{MHz}$ & $13.56 \mathrm{MHz}$ & $6.78 \mathrm{MHz}$ & $13.9 \mathrm{MHz}$ \\
\hline Efficiency & $91.5 \%$ & $84 \%$ & $84 \%$ & $90.1 \%$ & $94 \%$ & $72.6 \%$ & $0.39 \%$ \\
\hline Description & $\begin{array}{c}\text { Full CMOS } \\
\text { active } \\
\text { rectifier }\end{array}$ & $\begin{array}{c}\text { Full bridge } \\
\text { rectifier }\end{array}$ & $\begin{array}{c}\text { Class-E } \\
\text { current- } \\
\text { driven } \\
\text { rectifier }\end{array}$ & $\begin{array}{c}\text { Full wave } \\
\text { active } \\
\text { rectifier }\end{array}$ & $\begin{array}{c}\text { Passive } \\
\text { rectifier }\end{array}$ & $\begin{array}{c}\text { Reconfigurable } \\
\text { Rectifier }\end{array}$ & $\begin{array}{c}\text { Tissue- } \\
\text { Channel }\end{array}$ \\
\hline Power & $10 \mathrm{~W}$ & $10 \mathrm{~W}$ & $20 \mathrm{~W}$ & $10 \mathrm{~mW}$ & $3.2 \mathrm{~kW}$ & NA & NA \\
\hline
\end{tabular}

Modeling, characterization, and feasibility studies of capacitive coupling for power delivery and data communication have been performed in [26-32]. In [32], a near-field capacitive coupling-based wireless powering scheme in the subGHz frequency range is presented, where it could safely deliver up to $100 \mathrm{~mW}$ of power to an implant with a peak 
operating efficiency of over 50\%. In [33], Lee et al. proposed a voltage-boosted currentmode wireless power receiver for directly charging a low-voltage battery in implantable medical systems, where the technique is studied to charge low-voltage batteries wirelessly for supplying medical implantable systems. In [34], an inductive voltage/current mode integrated power management with seamless mode transition and energy recycling is proposed for robust inductive power delivery, based on parasitic bulk diodes of lower voltage drop.

The authors in [35-43] presented several novel circuit techniques to improve conversion efficiency of the rectification for MHz-range coupling. A design including both voltageand current-mode operation covering a wide range of coupling ratios between the coils is proposed in [34]. The so-called dual-loop adaptive delay compensation, voltage mode switched offset comparator, single-stage regulating, dynamically controllable comparator, adaptive delay time control, and single-stage AC-DC converter techniques have been proposed to achieve better active rectification operating at $13.56 \mathrm{MHz}$ [36-41]. In [44], an integrated resonant regulating rectifier has been presented for 144-MHz RF inputs ranging from 0.98 to $1.5 \mathrm{~V}$, where rectification and regulation is combined in a single stage.

A method to transmit power and to communicate data in the reverse direction over only one pair of inductive coils is described in [45]. The authors in [46] demonstrate that robust ultrasonic power-up and data uplink are able to be realized for implantable applications. A high-efficiency ultra-low-power CMOS rectifier is presented in [47] for RFpowered wearable medical devices, where a novel self-compensated cross-coupled design is proposed and realized. In [48], a new method is proposed for compensating coupling factor variations between transmitting and receiving coils for inductive power transfer. The papers in $[49,50]$ concern data transmission over inductive link, where state-of-the-art modulation/demodulation techniques are presented.

\subsection{PCE Estimation Model for the Active Full-Wave Rectifier}

Figure 7 shows an active full-wave rectifier power-conversion model used to estimate the PCE. In the figure, $\mathrm{C}_{\mathrm{g}, \mathrm{p}}$ is capacitance at the active diode capacitor gate terminal, and $R_{S}$ is output resistance when the active diode conduction is turned on. $R_{L}$ and $C_{L}$ are the output resistor and storage capacitor, respectively. The amount of charge is calculated using Equation (2) below, while the charge consumption is calculated using Equation (3), where $I_{R_{S}}$ and $I_{R_{L}}$ are, respectively, the current flowing through $R_{S}$ and $R_{L}$, and $D$ is the conduction duty cycle. Note that in the full-wave rectifier, a full cycle includes a single positive and negative half-cycle, thus $\mathrm{D}$ must be multiplied by two.

$$
\begin{gathered}
\mathrm{Q}_{\mathrm{ch}}=\mathrm{C}_{\mathrm{L}} \times \Delta \mathrm{V}=\left(\mathrm{I}_{\mathrm{R}_{\mathrm{S}}}-\mathrm{I}_{\mathrm{R}_{\mathrm{L}}}\right) \times 2 \times \mathrm{D} \times \mathrm{T}_{\mathrm{S}} \\
\mathrm{Q}_{\text {dis }}=\mathrm{C}_{\mathrm{L}} \times \Delta \mathrm{V}=\mathrm{I}_{\mathrm{R}_{\mathrm{L}}} \times(1-2 \mathrm{D}) \times \mathrm{T}_{\mathrm{S}}
\end{gathered}
$$

According to the conservation of charge and discharge, we can obtain the $\mathrm{I}_{\mathrm{R}_{\mathrm{S}}}$ relationship as follows:

$$
\mathrm{Q}_{\text {ch }}=\mathrm{Q}_{\text {dis }} \rightarrow \mathrm{I}_{\mathrm{R}_{\mathrm{S}}}=\frac{1}{2 \mathrm{D}} \mathrm{I}_{\mathrm{R}_{\mathrm{L}}}=\frac{1}{2 \mathrm{D}} \frac{\mathrm{V}_{\mathrm{REC}}}{\mathrm{R}_{\mathrm{L}}}
$$

We can then obtain all the parameters needed to calculate PCE. First, the PCE formula is as follows. $P_{\text {Load }}$ is the power consumption for output. That is, the output power is the total power consumption for the entire AC-DC conversion system.

$$
\begin{gathered}
\text { PEC }=\frac{P_{\text {Load }}}{P_{\text {Load }}+2 \times\left(P_{\text {ron }, \mathrm{p}}+P_{\text {ron }, \mathrm{n}}+\mathrm{P}_{\text {swp }, \mathrm{cg}}+\mathrm{P}_{\text {comp }}\right)} \\
\mathrm{P}_{\text {Load }}=\frac{\mathrm{V}_{\mathrm{REC}}{ }^{2}}{\mathrm{R}_{\mathrm{L}}}
\end{gathered}
$$

where $P_{\text {ron, } p}$ and $P_{\text {ron, } n}$ are, respectively, the power consumption of the active diode and the cross-couple and are calculated using Equations (7) and (8). Because the transistor 
operation conduction time is in the deep triode region, the transistor parameters can be used to calculate on-resistance using Equation (9). The switching power dissipation $\mathrm{P}_{\text {swp,cg }}$ at the transistor gate can be calculated using Equation (10), where the remaining $P_{\text {comp }}$ in Equation (5) is the power dissipation of the comparator and other auxiliary circuits.

$$
\begin{aligned}
& \mathrm{P}_{\text {ron }, \mathrm{p}}=\mathrm{I}_{\mathrm{R}_{\mathrm{S}}}{ }^{2} \times \mathrm{D} \times \mathrm{R}_{\mathrm{on}, \mathrm{p}} \\
&=\left(\frac{1}{2 \mathrm{D}} \frac{\mathrm{V}_{\mathrm{REC}}}{\mathrm{R}_{\mathrm{L}}}\right)^{2} \times \mathrm{D} \times \mathrm{R}_{\mathrm{on}, \mathrm{p}} \\
&= \frac{1}{4 \mathrm{D}}\left(\frac{\mathrm{V}_{\mathrm{REC}}}{\mathrm{R}_{\mathrm{L}}}\right)^{2} \times \mathrm{R}_{\mathrm{on}, \mathrm{p}} \\
& \mathrm{R}_{\mathrm{ron}, \mathrm{n}}= \frac{1}{4 \mathrm{D}}\left(\frac{\mathrm{V}_{\mathrm{REC}}}{\mathrm{R}_{\mathrm{L}}}\right)^{2} \times \mathrm{R}_{\mathrm{on}, \mathrm{n}} \\
& \mathrm{R}_{\mathrm{on}}= \frac{1}{\mu \mathrm{C}_{\mathrm{ox}} \frac{\mathrm{W}}{\mathrm{L}}\left(\mathrm{V}_{\mathrm{GS}}-\mathrm{V}_{\mathrm{TH}}\right)} \\
& \mathrm{P}_{\mathrm{swp}, \mathrm{cg}}=\mathrm{C}_{\mathrm{g}, \mathrm{p}} \times \mathrm{V}_{\mathrm{REC}}{ }^{2} \times \mathrm{f}_{\mathrm{s}}
\end{aligned}
$$

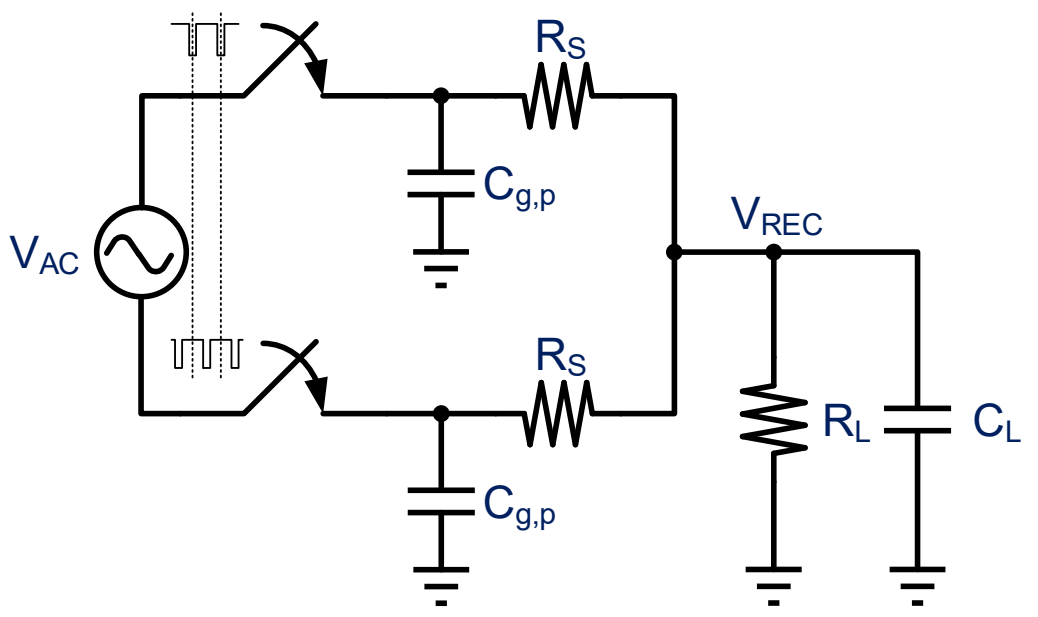

Figure 7. Calculation model for the active full-wave rectifier.

\section{Conclusions and Discussion}

In this paper, we mainly detail the wireless energy transfer applications involved from a circuit point of view and the existing developments in the rectification technology, where the foundation of wireless powering of energy harvesting for medical applications has also been introduced. The preceding architecture review shows that active rectifiers provide better energy conservation than passive rectifiers, but the passive architecture is not necessarily obsolete. Active diodes consume power for the comparator and compensation circuits, typically from 10 s to 100 s of $\mu \mathrm{W}$, with additional switching power dissipation from hundreds of $\mu \mathrm{W}$ to several $\mathrm{mW}$, confirming that given low input terminal energy $(<1 \mathrm{~mW})$, the active diode alone may not have sufficient power, and thus the rectifier may be unable to provide good PCE.

Therefore, an architecture should be selected based on the application scope. When the input source energy is lower than the $\mathrm{mW}$ level, hybrid passive rectifiers provide a practical solution, despite incurring a $\mathrm{V}_{\text {th }}$ pressure drop. This architecture is still capable of performing normal AC-DC energy conversion, and most digital circuits do not function properly without sufficient input power. On the other hand, higher input source energy levels offer more architecture options. In the field of biomedical applications, output power is usually below $100 \mathrm{~mW}$, which means the input power must be above $100 \mathrm{~mW}$, and an internal circuit power consumption of several $\mathrm{mW}$ is still within the acceptable range, making such applications suitable to use active diode architectures, such as a comparator. 
However, when input/output power reaches several watts, the size of the active diode grows, and the delay caused by the driver exceeds the comparator's ability to compensate. For active diodes, most high-power rectifiers use a voltage-controlled delay line (VCDL) to directly adjust the timing of active diode switching. While such circuits consume up to $10 \mathrm{~s}$ of $\mathrm{mW}$ of power, this is negligible for applications with inputs/outputs measuring several watts, so we can classify the architecture by application level. Despite the fact that active rectifier structures are able to provide higher voltage conversion efficiency compared with the passive designs demonstrated in the literature, their power conversion efficiency may be compromised due to the designer's skills.

Care must be taken to ensure that both the efficiency and hardware cost are excellent, otherwise it becomes meaningless to adopt such architectures. In general, the design of the rectifier first needs to consider the application specifications to determine the semiconductor process best suited, such as near-filed coupling, far-field coupling, or if it will be used for high-power biomedical applications (e.g., implants that require electrical stimulation) and so on.

In addition, it is necessary to consider whether the battery can be placed in the body for charging, or whether the operation of the implanted chip in the body can only be carried out by means of wireless power delivery. Moreover, the relative distance between the external platform and internal chips, such as whether it is easy to produce displacement affecting the difficulty of rectifier design, is also of primary importance. If the internal data must be sent back through the same rectifier for structure simplicity, the design will be more complicated.

Therefore, after the semiconductor process is determined for specific specifications, in addition to the impedance matching of the target load and the receiving coil, it is also necessary to pay attention to whether the implanted chip that receives power at the downstream stage has specific requirements for the power supply quality of the rectifier output. Last but not least, the system developer should also pay attention to the limitations caused by the reduced complexity of the design itself, after simplifying the rectifier circuit, to see if the desired conversion efficiency can be achieved under compromise.

Future research and development trends for the entire range of applications focus on finding ways to reduce the countercurrent and circuit power consumption in order to continuously reduce consumption to levels of several $\mu \mathrm{W}$, allowing for low-voltage active rectifiers of high-efficiency energy harvesting.

Funding: This research was funded by MINISTRY OF SCIENCE AND TECHNOLOGY (MOST) grant numbers 109-2221-E-182-036 and 110-2221-E-182-053. And The APC was funded by CHANG GUNG MEMORIAL HOSPITAL (CGMH).

Acknowledgments: The authors would like to thank Shiang-Wei Li, Ta-Hao Kuo, Hsiang-Chi Cheng, Shiang-Wei Li, Yi-Zhong Huang, Gui-Hong Zeng, and Zheng-Lin Chen for their technical assistance. This study was funded in part with grant from the MINISTRY OF SCIENCE AND TECHNOLOGY (MOST), Taiwan, under Grants MOST 109-2221-E-182-036 and 110-2221-E-182-053. This work is also supported, in part, by the CHANG GUNG MEMORIAL HOSPITAL (CGMH) under Contract CMRPD2L0031.

Conflicts of Interest: The author declares no competing financial interests.

\section{References}

1. Sauer, C.; Stanacevic, M.; CauWenberghs, G.; Thakor, N. Power harvesting andtelemetryin CMOS for implanted devices. IEEE Trans. Circuits Syst. I 2005, 52, 2605-2613. [CrossRef]

2. Georgiou, J.; Toumazou, C. A 126 W cochlear chip for a totally implantable system. IEEE J. Solid-State Circuits 2005, 40, 430-443. [CrossRef]

3. Sivaprakasam, M.; Liu, W.; Wang, G.; Weiland, J.D.; Humayun, M.S. Architecture tradeoffs in high-density microstimulators for retinal prosthesis. IEEE Trans. Circuits Syst. I 2005, 52, 2629-2641. [CrossRef]

4. Lee, S.Y.; Lee, S.C. An implantable wireless bidirectional communication microstimulator for neuromuscular stimulation. IEEE Trans. Circuits Syst. I 2005, 52, 2526-2538. 
5. Ghovanloo, M.; Najafi, K. Fully integrated wideband high-current rectifiers for inductively powered devices. IEEE J. Solid-State Circuits 2004, 39, 1976-1984. [CrossRef]

6. Hashemi, S.; Sawan, M.; Savaria, Y. A novel low-drop CMOS active rectifier for RF-powered devices: Experimental results. J. Microelectron. 2009, 40, 1547-1554. [CrossRef]

7. Zargham, M.; Glenn Gulak, P. High-efficiency CMOS rectifier for fully integrated $\mathrm{mW}$ wireless power transfer. In Proceedings of the 2012 IEEE International Symposium on Circuits and Systems (ISCAS), Seoul, Korea, 20-23 May 2012; pp. $2869-2872$.

8. Saeid Hashemi, S.; Sawan, M.; Savaria, Y. A high-efficiency low-voltage CMOS rectifier for harvesting energy in implantable devices. IEEE Trans. Biomed. Circuits Syst. 2012, 6, 326-335. [CrossRef]

9. Chen, C.L.; Chen, K.H.; Liu, S.I. Efficiency-enhanced CMOS rectifier for wireless telemetry. IET Electron. Lett. 2007, 43, 976-978. [CrossRef]

10. Peters, C.; Handwerker, J.; Maurath, D.; Manoli, Y. A sub-500 mV highly efficient active rectifier for energy harvesting applications. IEEE Trans. Circuits Syst. I 2011, 58, 1542-1550. [CrossRef]

11. Lee, H.M.; Ghovanloo, M. An integrated power-efficient active rectifier with offset-controlled high speed comparators for inductively powered applications. IEEE Trans. Circuits Syst. I 2011, 58, 1749-1760. [CrossRef]

12. Heng, L.; Ki, W.H.; Lu, Y.; Yim, T.S. Adaptive on/off delay-compensated active rectifiers for wireless power transfer systems IEEE J. Solid-State Circuits 2016, 51, 712-723.

13. Park, H.G.; Jang, J.H.; Kim, H.-J.; Park, Y.-J.; Oh, S.J.; Pu, Y.G.; Hwang, K.C.; Yang, Y.; Lee, K.Y. A Design of a Wireless Power Receiving Unit with a High-Efficiency 6.78-MHz Active Rectifier Using Shared DLLs for Magnetic-Resonant WP Applications. IEEE Trans. Power Electron. 2016, 31, 4484-4498. [CrossRef]

14. Fu, M.F.; Tang, Z.F.; Liu, M.; Ma, C.B.; Zhu, X.N. Full-Bridge Rectifier Input Reactance Compensation in Megahertz Wireless Power Transfer Systems. In Proceedings of the 2015 IEEE PELS Workshop on Emerging Technologies: Wireless Power, Daejeon, Korea, 5-6 June 2015; pp. 1-5.

15. Liu, M.; Fu, M.F.; Ma, C.B. Parameter Design for a 6.78-MHz Wireless Power Transfer System Based on Analytical Derivation of Class E Current-Driver Rectifier. IEEE Trans. Power Electron. 2016, 31, 4280-4291. [CrossRef]

16. Lu, Y.; Ki, W.H. A $13.56 \mathrm{MHz}$ CMOS Active Rectifier with Switched-Offset and Compensated Biasing for Biomedical Wireless Power Transfer System. IEEE Trans Biomed Circuits Syst. 2013, 8, 334-344. [CrossRef] [PubMed]

17. Shamseh, M.B.; Yuzurihara, I.; Kawamura, A. A 3.2-kw 13.56-MHz SiC Passive Rectifier With 94\% Efficiency Using Commutation Capacitor. IEEE Trans. Power Electron. 2016, 31, 6787-6791. [CrossRef]

18. Liu, Z.T.; Zhong, Z.; Guo, Y.X. In Vivo High-Efficiency Wireless Power Transfer with Multisine Excitation. IEEE Trans. Microw. Theory Tech. 2017, 65, 3530-3540. [CrossRef]

19. Chen, P.P.; Yang, H.Z.; Luo, R.; Zhao, B. A Tissue-Channel Transcutaneous Power Transfer Technique for Implantable Devices IEEE Trans. Power Electron. 2018, 33, 9753-9761. [CrossRef]

20. Zeng, Q.; Zhao, S.; Yang, H.; Zhang, Y.; Wu, T. Micro/Nano Technologies for High-Density Retinal Implant. Micromachines 2019, 10, 419. [CrossRef] [PubMed]

21. Gagnon-Turcotte, G.; Khiarak, M.N.; Ethier, G.; Koninck, Y.D.; Gosselin, B. A $0.13 \mu \mathrm{m}$ CMOS SoC for simultaneous multichannel optogenetics and electrophysiological brain recording. IEEE J. Solid-State Circuits 2018, 53, 3087-3100. [CrossRef]

22. Lu, C.; Tsui, C.Y.; Ki, W.-H. Vibration Energy Scavenging System with Maximum Power Tracking for Micropower Applications. IEEE Trans. Very Large Scale Integr. (VLSI) Syst. 2011, 19, 2109-2119. [CrossRef]

23. Raghunathan, V. Design and power management of energy harvesting embedded systems. In Proceedings of the 2006 International Symposium on Low Po wer Electronics and Design, Tegernsee, Germany, 4-6 October 2006; pp. 369-374.

24. Lu, C.; Raghunathan, V.; Roy, K. Efficient Design of Micro-Scale Energy Harvesting Systems. IEEE J. Emerg. Sel. Top. Circuits Syst. 2011, 1, 254-266. [CrossRef]

25. Chen, C.Y.; Chou, P.H. DuraCap: A supercapacitor-based, power-bootstrapping, maximum power point tracking energyharvesting system. In Proceedings of the 2010 ACM/IEEE International Symposium on Low-Power Electronics and Design, Austin, TX, USA, 18-20 August 2010; pp. 313-318.

26. Zhang, K.; Hao, Q.; Song, Y.; Wang, J.; Huang, R.; Liu, Y. Modeling and characterization of the implant intra-body communication based on capacitive coupling using a transfer function method. Sensors 2014, 14, 1740-1756. [CrossRef]

27. Sawma, C.; Sawan, M.; Kassem, A. Capacitive data links intended for implantable medical devices: A survey. In Proceedings of the 2015 International Conference on Advances in Biomedical Engineering (ICABME), Beirut, Lebanon, 16-18 September 2015; pp. 266-269.

28. Erfani, R.; Marefat, F.; Mohseni, P. Biosafety considerations of a capacitive link for wireless power transfer to biomedical implants. In Proceedings of the 2018 IEEE Biomedical Circuits and Systems Conference (BioCAS), Cleveland, OH, USA, 17-19 October 2018; pp. 355-358.

29. Erfani, R.; Marefat, F.; Sodagar, A.M.; Mohseni, P. Transcutaneous capacitive wireless power transfer (C-WPT) for biomedical implants. In Proceedings of the 2017 IEEE International Symposium on Circuits and Systems (ISCAS), Baltimore, MD, USA, 28-31 May 2017; pp. 2561-2564.

30. Erfani, R.; Marefat, F.; Sodagar, A.M.; Mohseni, P. Modeling and characterization of capacitive elements with tissue as dielectric material for wireless powering of neural implants. IEEE Trans. Neural Syst. Rehabil. Eng. 2018, 26, 1093-1099. [CrossRef] [PubMed] 
31. Koruprolu, A.; Nag, S.; Erfani, R.; Mohseni, P. Capacitive wireless power and data transfer for implantable medical devices. In Proceedings of the 2018 IEEE Biomedical Circuits and Systems Conference (BioCAS), Cleveland, OH, USA, 17-19 October 2018; pp. 291-294.

32. Jegadeesan, R.; Agarwal, K.; Guo, Y.-X.; Yen, S.-C.; Thakor, N.V. Wireless power delivery to flexible subcutaneous implants using capacitive coupling. IEEE Trans. Microw. Theory Tech. 2017, 65, 280-292. [CrossRef]

33. Lee, S.-H.; Bang, J.-S.; Yoon, K.-S.; Gwon, H.-D.; Kim, S.-W.; Cho, I.-K.; Hong, S.-W.; Cho, G.H. Voltage-boosted current-mode wireless power receiver for directly charging a low-voltage battery in implantable medical systems. IEEE Trans. Ind. Electron. 2019, 66, 8860-8865. [CrossRef]

34. Gougheri, H.S.; Kiani, M. An inductive voltage-/currentmode integrated power management with seamless mode transition and energy recycling. IEEE J. Solid-State Circuits 2019, 54, 874-884. [CrossRef]

35. Engur, Y.; Ulusan, H.; Yigit, H.A.; Chamanian, S.; Kulah, H. $13.56 \mathrm{MHz}$ mixed mode rectifier circuit for implantable medical devices. In Proceedings of the 19th International Conference on Micro and Nanotechnology for Power Generation and Energy Conversion Applications (PowerMEMS), Krakow, Poland, 2-6 December 2019; pp. 1-4.

36. Erfani, R.; Marefat, F.; Nag, S.; Mohseni, P. A 1-10-MHz frequency-aware CMOS active rectifier with dual-loop adaptive delay compensation and $>230-\mathrm{mW}$ output power for capacitively powered biomedical implants. IEEE J. Solid-State Circuits 2020, 55, 756-766. [CrossRef]

37. Noh, K.; Amanor-Boadu, J.; Zhang, M.; Sanchez-Sinencio, E. A 13.56-MHz CMOS active rectifier with a voltage mode switched offset comparator for implantable medical devices. IEEE Trans. Very Large Scale Integr. Syst. 2018, 26, 2050-2060. [CrossRef]

38. Huang, C.; Kawajiri, T.; Ishikuro, H. A 13.56-MHz wireless power transfer system with enhanced load-transient response and efficiency by fully integrated wireless constant-idle-time control for biomedical implants. IEEE J. Solid-State Circuits 2018, 53, 538-551. [CrossRef]

39. Cheng, H.-C.; Gong, C.-S.A.; Kao, S.-K. A 13.56 MHz CMOS high-efficiency active rectifier with dynamically controllable comparator for biomedical wireless power transfer systems. IEEE Access 2018, 6, 49979-49989. [CrossRef]

40. Xue, Z.; Fan, S.; Li, D.; Zhang, L.; Gou, W.; Geng, L. A 13.56 MHz, 94.1\% peak efficiency CMOS active rectifier with adaptive delay time control for wireless power transmission systems. IEEE J. Solid-State Circuits 2019, 54, 1744-1754. [CrossRef]

41. Xue, Z.; Fan, S.; Zhang, L.; Guo, Z.; Dong, L.; Li, D.; Geng, L. Single-stage dual-output AC-DC converter for wireless power transmission. In Proceedings of the 2018 IEEE Custom Integrated Circuits Conference (CICC), San Diego, CA, USA, 8-11 April 2018; pp. 1-4.

42. Cheng, L.; Ki, W.-H.; Tsui, C.-Y. A 6.78-MHz single-stage wireless power receiver using a 3-mode reconfigurable resonant regulating rectifier. IEEE J. Solid-State Circuits 2017, 52, 1412-1423. [CrossRef]

43. Mohammadi, A.; Redoute, J.; Yuce, M.R. Wireless power transmission for biomedical implants: The role of near-zero threshold CMOS rectifiers. In Proceedings of the 37th Annual International Conference of the IEEE Engineering in Medicine and Biology Society (EMBC), Milan, Italy, 25-29 August 2015; pp. 5453-5456. [CrossRef]

44. Kim, C.; Ha, S.; Park, J.; Akinin, A.; Mercier, P.P.; Cauwenberghs, G. A 144-MHz fully integrated resonant regulating rectifier with hybrid pulse modulation for mm-sized implants. IEEE J. Solid-State Circuits 2017, 52, 3043-3055. [CrossRef]

45. Pan, J.; Abidi, A.A.; Jiang, W.; Markovic, D. Simultaneous transmission of up to 94-mW self-regulated wireless power and up to $5-\mathrm{Mb} / \mathrm{s}$ reverse data over a single pair of coils. IEEE J. Solid-State Circuits 2019, 54, 1003-1016. [CrossRef]

46. Weber, M.J.; Yoshihara, Y.; Sawaby, A.; Charthad, J.; Chang, T.C.; Arbabian, A. A miniaturized single-transducer implantable pressure sensor with time-multiplexed ultrasonic data and power links. IEEE J. Solid-State Circuits 2018, 53, 1089-1101. [CrossRef]

47. Noghabaei, S.M.; Radin, R.L.; Savaria, Y.; Sawan, M. A High-Efficiency Ultra-Low-Power CMOS Rectifier for RF Energy Harvesting Applications. In Proceedings of the 2018 IEEE International Symposium on Circuits and Systems (ISCAS), Florence, Italy, 27-30 May 2018.

48. Trigui, A.; Hached, S.; Mounaim, F.; Ammari, A.C.; Sawan, M. Inductive Power Transfer System with Self-Calibrated Primary Resonant Frequency. IEEE Trans. Power Electron. 2015, 30, 6078-6087. [CrossRef]

49. Trigui, A.; Ali, M.; Ammari, A.C.; Savaria, Y.; Sawan, M. Energy Efficient Generic Demodulator for High Data Transmission Rate Over an Inductive Link for Implantable Devices. IEEE Access 2019, 7, 159379-159389. [CrossRef]

50. Trigui, A.; Ali, M.; Hached, S.; David, J.-P.; Ammari, A.C.; Savaria, Y.; Sawan, M. Generic Wireless Power Transfer and Data Communication System Based on a Novel Modulation Technique. IEEE Trans. Circuits Syst. I Regul. Pap. 2020, 67, 3978-3990. [CrossRef] 